\title{
Photoluminescence from a soluble semiconducting polymer in waveguide and microcavity configurations
}

\author{
M. Allegrini a, A. Arena ${ }^{\text {a }}$, M. Labardi ${ }^{\text {b }}$, G. Martino a, R. Girlanda a , C. Pace ${ }^{\text {a }}$, \\ S. Patanè ${ }^{a}$, G. Saitta ${ }^{a, *}$, S. Savasta ${ }^{\text {a }}$ \\ a Dipartimento di Fisica della Materia e Tecnologie Fisiche Avanzate, Salita Sperone 31, Istituto Nazionale per la Fisica della Materia \\ (INFM), Università di Messina, I-98160 Sant'Agata-Messina, Italy \\ ${ }^{b}$ Istituto Nazionale per la Fisica della Materia (INFM), Università di Pisa, Italy
}

\begin{abstract}
The optical properties of thin films of PVK- $\mathrm{Alq}_{3}$ are examined by means of spectrophotometry in the UV-VIS-NIR. Photoluminescence (PL) measurements indicate that the polymer films deposited on glass substrates behave as asymmetrical planar waveguides for the emitted light. The waveguided luminescence shrinks and change color with respect to the non-waveguided emission. Stronger changes in the luminescence features are observed using a microcavity configuration, obtained by spin-coating PVK-Alq ${ }_{3}$ on an inorganic Distributed Bragg Reflector (DBR) and by vacuum-depositing a thick aluminum layer on the top of the polymer film.
\end{abstract}

Keywords: Polymer semiconductors; Organic-inorganic microcavities

\section{Introduction}

In the last few years organic semiconducting polymers have attracted a great deal of attention owing to their potential use as active layers in solidstate lasers and polychromatic light emitting diodes [1-4]. Here we report on the optical properties of PVK-Alq ${ }_{3}$, a soluble material obtained by electrochemically blending poly(9-vinylcarbazole) (PVK), a transparent hole-semiconducting polymer, with alu-

\footnotetext{
Corresponding author. Telefax: +39-90-391382; E-mail saitta@ortica.unime.it
}

minum III-hydroxyquinoline $\left(\mathrm{Alq}_{3}\right)$, an organic electron-semiconductor, characterized by a green luminescence with radiative yield of 0.45 , when excited with UV or blue light [5]. Films of PVK-Alq doped with a substituted Coumarin, whose thickness ranges between some tens and several hundreds of nanometers, are easily obtained by spin-coating 1,1,2-trichloroethane solutions of $\mathrm{PVK}-\mathrm{Alq}_{3}$ added with a $1 \%$ by weight of Coumarin.

In the present paper we examine how the photoluminescence (PL) lineshape of PVK-Alq 3 can be easily engineered, by adopting suitable device configurations. In particular we show that owing to a waveguiding effect at the air-polymer-glass inter- 
faces, the luminescence color can be changed from blue to red simply by acting on the thickness of PVK-Alq ${ }_{3}$ films. Then we investigate on the modification of the PL lineshape that are observed by sandwiching $\mathrm{PVK}-\mathrm{Alq}_{3}$ between a high reflectance inorganic Distributed Bragg Reflector (DBR) and an aluminum mirror.

\section{Experimental}

The PVK-Alq ${ }_{3}$ films show an intense green emission under UV or blue light irradiation, are air and moisture insensitive, do not show any ageing problem in several months and are photostable under prolonged exposition to a continuous $\mathrm{Ar}^{+}$laser line $\left(\lambda=459 \mathrm{~nm}, 24 \mathrm{~mW}\right.$, spotsize $\left.\sim 0.25 \mathrm{~mm}^{2}\right)$.

PL measurements are performed using a Perkin Elmer 650 fluorometer with a $150 \mathrm{~W}$ Xenon lamp. An interference filter is used to obtain an exciting beam centred at $365 \mathrm{~nm}$, with a FWHM of $20 \mathrm{~nm}$. The emitted light collection angle is allowed to vary by rotating the sample, a diaphragm is used to eliminate scattered light and a polarizer is positioned in front of the analyser.

The reflectivity measurements are carried out by means of a Perkin Elmer Lambda 2 spectrophotometer, equipped with a couple of reflectance accessories.

The films thickness is determined using a DEKTAK 3030 stylus micro-profilometer.

\section{Results and discussion}

The emission and excitation spectra of a film of PVK- $\mathrm{Alq}_{3}$, measured using a $150 \mathrm{~W}$ Xenon lamp as excitation are shown in Fig. 1. The same figure shows the results of absorption measurements performed on pure PVK, pure $\mathrm{Alq}_{3}$ and $\mathrm{PVK}-\mathrm{Alq}_{3}$. Comparing the absorption and the excitation profiles, one can notice the presence of two strong excitation peaks in the UV, ascribed to the occurrence of electronic transitions in the PVK matrix, and a broad band, centered at about $390 \mathrm{~nm}$, assigned to the $\mathrm{Alq}_{3}$ chromophore. The PL spectrum of PVK-Alq ${ }_{3}$ dis-
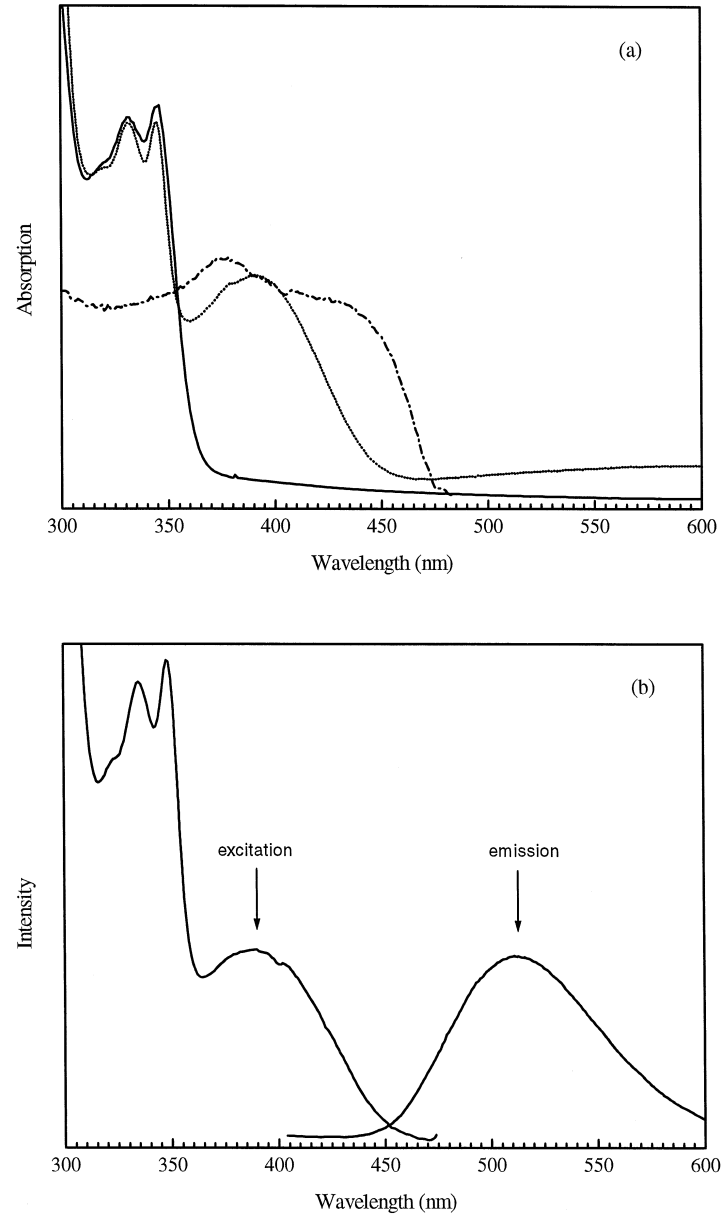

Fig. 1. (a) Absorption spectrum of a PVK film (solid), of the $\mathrm{Alq}_{3}$ powder (dashed-dotted) and of $\mathrm{PVK}-\mathrm{Alq}_{3}$ film on $\mathrm{KBr}$ substrate (dotted); (b) excitation and emission spectra of a Coumarin doped PVK-Alq ${ }_{3}$ film.

played in Fig. 1b, is collected by exciting with light of $365 \mathrm{~nm}$ and observing the emission at about $30^{\circ}$ out of the surface normal. The PL spectrum is centered at about $515 \mathrm{~nm}$, is unpolarized and has a FWHM of about $100 \mathrm{~nm}$. The behavior of the PL changes if one collects the emitted photons along the polymer edge, at $90^{\circ}$ out of the surface normal. In fact PVK-Alq ${ }_{3}$ films deposited on glass act as asymmetrical planar waveguides since the polymer index of refraction $(n=1.68$ at $568 \mathrm{~nm})$ is higher than those of the surrounding media (air, $n=1$ and glass, $n=1.5$ ). The waveguided PL profile and its depen- 
dence upon light polarization are strongly affected by the film thickness as can be observed in Fig. 2, where the waveguided emission spectra measured on three different films are displayed. One can notice
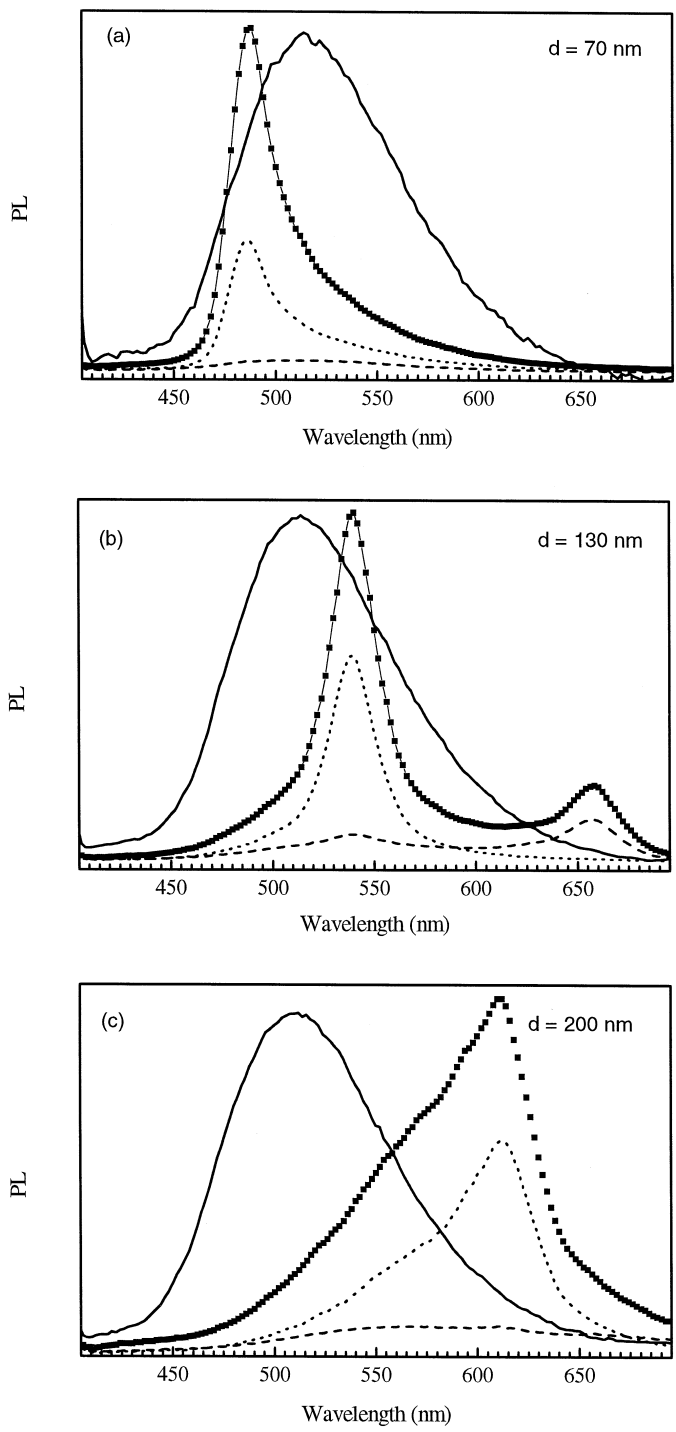

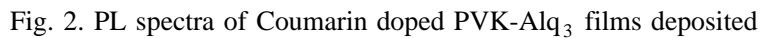
on glass substrates (the curves displayed in (a), (b) and (c) are obtained on 70, 130 and $200 \mathrm{~nm}$ thick films, respectively). Solid lines represent the non-waveguided PL, observed at about $30^{\circ}$ out of the normal. The scatter curves (squares) represent the waveguided PL spectra, the dashed and dotted lines describe the PL linearly polarized along directions at $90^{\circ}$ each other. that the waveguided emission of the $70 \mathrm{~nm}$ thick film, shown in Fig. 2a shifts by more than $30 \mathrm{~nm}$ towards the lower wavelength region with respect to the non-waveguided PL. The linewidth shrinks by more than the $70 \%$ and the emitted light is strongly linearly polarized. The waveguided luminescence of a 130 -nm thick film, displayed in Fig. 2b, is characterized by two peaks centered at about 540 and 658 $\mathrm{nm}$, sharpened with respect to the non-waveguided emission, each linearly polarized along two directions at $90^{\circ}$ each other. Fig. $2 \mathrm{c}$ shows the waveguided luminescence of a film whose thickness is $200 \mathrm{~nm}$. The spectrum exhibits a peak, positioned at about $615 \mathrm{~nm}$, superimposed on a broad band centered at approximately $570 \mathrm{~nm}$. Summarizing, the results shown in Fig. 2 indicate that a suitable tuning of the polymer film thickness, allows the waveguided luminescence wavelength maximum to be selected, obtaining linearly polarized emitted light whose color spans from the blue to the red. The experimental results shown here, and in particular the cut-off of the waveguided luminescence in the higher wavelength region observed in Fig. 2, are confirmed by the results of preliminary theoretical calculations on asymmetrical active waveguides performed by us. According to the literature [6-8], the decrease of the luminescence width in simple airpolymer-glass configuration is usually observed only using pulsed laser light above a certain energy threshold. Further increase in the power of the pump can induce processes such as amplified spontaneous emission, super-radiance, super-fluorescence or stimulated emission. The spectral narrowing observed using as excitation a Xenon lamp, therefore, is suggestive of the potentiality of the PVK-Alq ${ }_{3}$ films as active layer in optical devices.

As a further example of PL modifications that can be achieved by using structures that confine the electromagnetic field, we report the preliminary results obtained on a inorganic-organic microcavity. The device consists of a spin coated, $200 \mathrm{~nm}$ thick, PVK-Alq ${ }_{3}$ layer, sandwiched between an inorganic DBR and a $1-\mu \mathrm{m}$ thick Aluminum film. The DBR is a stack of seven couples of layers of $\mathrm{MgF}_{2}(n=1.35)$ and $\mathrm{ZrO}_{2}(n=2)$, followed by one more $\mathrm{MgF}_{2}$ layer, deposited on a BK7 glass substrate by laser induced physical vapor deposition [9]. The thickness of DBR layers is a quarter of wave at $520 \mathrm{~nm}$ and 


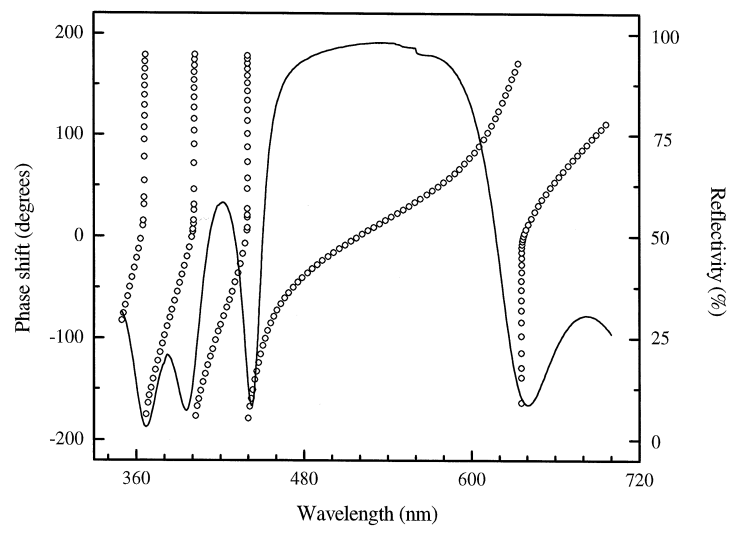

reflectivity of the DBR has its maximum, in correspondence of the dip observed in the microcavity reflectivity curve. The 505-nm luminescence peak is a cavity mode, and represents the resonance condition for the 200-nm thick PVK-Alq ${ }_{3}$ film. As can be observed from the mirror phase-shift behavior, this peak falls in a linear phase-shift region, where the resonance condition can easily be tuned by changing the polymer film thickness. As a further evidence of the resonant nature of the 505-nm peak, one can observe the characteristic dispersive behavior of the microcavity angle resolved PL curves, shown in Fig. 4. The PL spectra change strongly as a function of the viewing angle: correspondingly the emission color modifies from red to green as the observer moves from the surface normal.

The PL and $R$ curves also show two satellite resonance peaks corresponding to DBR reflectivity edges, where fast phase-shift changes occur; to avoid that, the design of a single peak microcavity would require DBR mirrors with slow-varying phase-shift profile over a wavelength region larger than the polymer emission band.

In conclusion we have shown that simple device design allows the spectral properties of emission of

Fig. 3. (a) Reflectivity spectrum (solid) and phase shift (open circles) of the dielectric mirror; (b) experimental reflectivity spectrum of the microcavity (solid) and PL of the microcavity at normal incidence (open circles).

the measured reflectivity is shown in Fig. 3a together with the calculated behavior of the phase-shift acquired by a wave reflected inside the cavity, at the polymer-DBR interface. At the polymer-aluminum interface the reflectivity and phase-shift are near constant ( $88 \%$ and $146^{\circ}$, respectively) over the wavelength region of interest. The microcavity is resonant at those wavelengths determined by the condition that the phase-shift is $2 \pi$ multiple after a complete round trip. Fig. $3 \mathrm{~b}$ shows the microcavity reflectivity $(R)$ and PL curves, measured along the surface normal $\left(\theta=0^{\circ}\right)$. The PL curve is characterized by a $10 \mathrm{~nm}$ wide peak, centered at $505 \mathrm{~nm}$, near the value of $520 \mathrm{~nm}$ for which the microcavity was designed. Such peak is positioned in the region where the

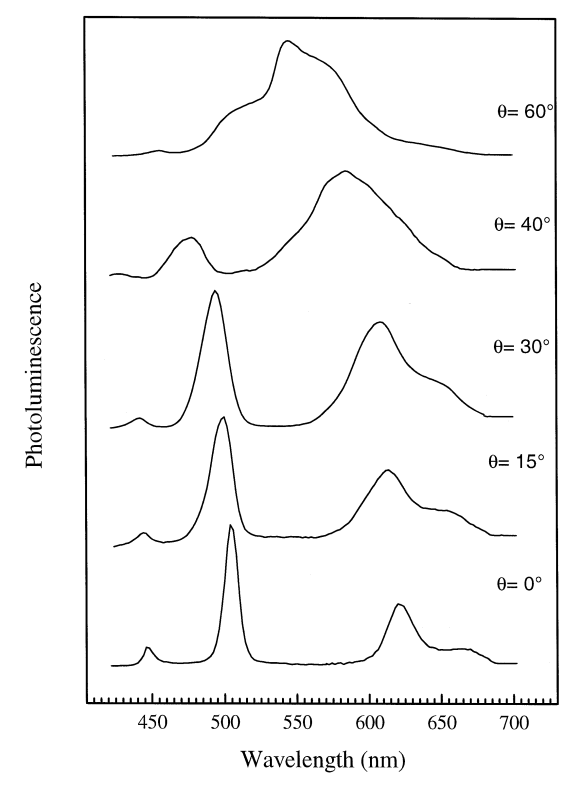

Fig. 4. PL spectra of the microcavity as a function of the viewing angle $\theta$. 
PVK-Alq ${ }_{3}$ to be controlled, obtaining light whose color covers the visible region from blue to red. The material here analyzed offers important advantages such as the semiconducting nature, the low cost and the high processability. Furthermore large area films can be easily obtained by spin-coating. The features of PVK-Alq ${ }_{3}$ and the easy of fabrication and high integrability of the simple structures discussed here, seem to be of great importance in view of obtaining optical or opto-electronic devices.

\section{Acknowledgements}

The authors gratefully acknowledge the Centro per la Ricerca Elettronica in Sicilia di Monreale (PA) for the technical and scientific support.

\section{References}

[1] V. Bulovic, G. Gu, P.E. Burrows, S.R. Forrest, M.E. Thompson, Nature 380 (1996) 29.

[2] J. Kalinowski, P. Di Marco, M. Cocchi, V. Fattori, N. Camaioni, J. Duff, Appl. Phys. Lett. 68 (1996) 2317.

[3] H. Becker, S.E. Burns, R.H. Friend, Phys. Rev. B 56 (1997) 1893.

[4] J. Greener, F. Cacialli, I.D.W. Samuel, R.H. Friend, Synth. Met. 76 (1996) 137.

[5] M. Allegrini, A. Arena, R. Girlanda, C. Pace, S. Patané, G. Saitta, submitted for publication on J. Mater. Res.

[6] V.G. Kozlov, V. Bulovic, P.E. Burrows, S.R. Forrest, Nature 389 (1997) 362.

[7] V. Bulovic, V.G. Kozlov, V. Bulovic, Khalfin, S.R. Forrest, Science 279 (1998) 553.

[8] M.A. Diaz-Garcia, F. Hide, B.J. Schwartz, M.D. McGehee, M.R. Andersson, A.J. Heeger, Appl. Phys. Lett. 70 (1997) 3191.

[9] C. Pace, E. Nava, C. Calì, Pure Appl. Opt. 3 (1994) 441. 